\title{
NEUROSIS AND SEXUALITY
}

\author{
BY
}

\section{ELIOT SLATER}

(RECEIVED 31st JANUARY, 1945)

\begin{abstract}
Introduction
Neurotic men have been found to differ from normal men in a number of particulars, in the frequency with which they show qualitative findings and in the degree to which they show quantitative findings. In addition they have been found to be abnormally heterogeneous. In respect of graded characters we may expect them at times to vary from normals at both ends of the scale. If we choose a single criterion for the distinguishing of neurotics from normals, we cannot expect it to be of value over more than part of the range; it may be useful in distinguishing some neurotics but not all. For instance, Rees and Eysenck (1945) have shown that an index relating stature to transverse chest diameter (1) exhibited greater variability in the neurotic than in the normal, (2) was associated at one end of the scale with symptoms of anxiety, depression, effort syndrome, etc., (3) at the other extreme was associated with hysterical symptoms. Attention is rightly being paid to the physical correlates of psychological qualities. For physical characteristics are often capable of preciser definition and more exact measurement than any psychological characteristic with which they may be associated; and the discovery of an association between a physical and mental trait may give us a clue to causal relationship. For this purpose it is desirable to examine the neurotic and the normal subject particularly in respect of those physical and psychological qualities where deviations might be expected to have an effect in reducing good health, adaptability, or emotional stability.
\end{abstract}

In the psycho-analytic theory of the development of the neuroses it has always been maintained that the eventual neurotic adaptation of the adult is determined by disturbances of one kind or another in early psycho-sexual development. This theory has been supported by a wealth of individual observations, but by no general evidence that the sexual activity of neurotics differs in any way from that of the normal. A certain amount of evidence of this kind has, however, been forthcoming from other sources. Billings et al. (1943) made a comparison between 100 U.S. Army psychiatric patients and 100 enlisted men. Of the psychiatric patients 54 were neurotic or showed psychopathic personality, while the remainder showed schizophrenic or affective symptoms, or were feeble-minded.
They state that 44 per cent. more of the enlisted men than of the patients showed an average sexual development, 30 per cent. had regular girl friends 41 per cent. more had intentions to marry. Absolute figures are not given. Also in the U.S.A. Steinberg and Wittman (1943) have reported on their findings on 267 men from the Services: Of these 158 formed a control group, 22 were predominantly neurotic, and 87 predominantly psychotic. As tested by the Elgin developmental history, the psychotic subjects (of whom 76 per cent. were schizophrenic) made a less satisfactory early sex adjustment than the controls, but the difference between the neurotic patients and the controls was negligible; the same was largely true for adult sex adjustment. As far as the female is concerned, it is worth noting that Bernard (1935), working with a group of married couples, found significant positive correlation coefficients between (1) marital dissatisfaction of wife and neuroticism of wife, (2) marital dissatisfaction of husband and neuroticism of wife, and (3) days since last coitus and neuroticism of wife; there was, however, no significant correlation between days since last coitus and neuroticism of husband. It cannot be asserted that any of this evidence is very precise or takes us very far.

In a previous communication (Slater, 1943), dealing with the statistical analysis of the clinical records of two thousand neurotic soldiers, it was observed that a considerable proportion (45 per cent.) had led lives in which sexual activity had played a surprisingly minor rôle. In these men " sexual relations began at a late age, occurred with unusual rarity and were associated with little interest or satisfaction." The question whether the sexual activity of an individual patient was to be regarded as inadequate or normal in degree was decided by the doctor in charge of the case. No doubt standards of judgment varied from clinician to clinician. But as each doctor would be consistent with his own standard, the division of the case material into two classes in this way does represent a real though crude distinction. From here on the one group will be referred to as "inadequate" and the other as " average" in respect of sex life. The statistical material was analysed, using this criterion for classification, but consideration of the findings was postponed as it was hoped to combine the data 
with those obtained from a biochemical and genetical investigation which was then in progress. Unfortunately, these latter investigations had to be interrupted, and are not likely to be completed in the near future. The results of the statistical investigation are, however, so interesting that it is thought that they merit publication on their own account. In the table below are listed all and only those findings in which there was a statistically significant difference between the frequencies observed in the two groups of men with " inadequate" and with " average" sex lives."

\section{Results}

Several interesting points appear from the table. The first is that the group of under-sexed men are clearly a more abnormal group than the others. In them, with few exceptions, the positive psychiatric findings are more frequent. The exceptions are themselves interesting. Head injury is more frequent in the group of men with " average " sexuality; it is a finding which has already been shown (Slater, 1943) to be associated with lesser rather than greater degrees of constitutional predisposition. The amnesic symptoms are also more frequent in the averagely sexed group; these symptoms are particularly frequent among men who break down in battle (Sargant and Slater, 1941). The only abnormal personality trait which is more frequent among the averagely sexed are the qualities of impulsiveness or aggressiveness. This can be related to the finding by Denis Hill that patients suffering from the clinical electro-encephalographic syndrome which he has termed "dysrhythmic aggressive behaviour" (Hill, 1944) are very commonly troubled by a quite excessive libide (personal communication).

The second point of interest is that where a graded characteristic is being considered, the sexually inadequate tend to be found in excess at one or other extreme. Compared with men of average sexuality, they are more likely to have either a poor or a good school record, to be either of poor or of good intelligence, and to be relatively infrequently average in these respects. They are more likely to have an asthenic or a pyknic physique and are less likely to be of athletic habitus. Exactly parallel findings have recently been reported from comparisons of neurotics as a whole with normal controls. Eysenck (1943) has found that neurotics were less frequently average in respect of intelligence, and Rees and Eysenck (1945) have found that they were less frequently average in respect of bodily habitus than were the normal men with whom they were compared.

\footnotetext{
1 It is to be noted that there was a small but significant difference between the mean ages of the "inadequate " and "average "groups. This indicated that the clinicians, in forming their judgment, had not been able to allow sufficiently for the effects of age. For the young must of necessity have had as a rule a less abundant sex life than their seniors. It was therefore thought desirable to make allowance for this adventitious factor. The necessary corrections for age, which all proved to be very small, have therefore been made throughout the table, a process which though laborious was quite simple as the material had been classified by age groups as well as by sex life in the primary card counts.
}

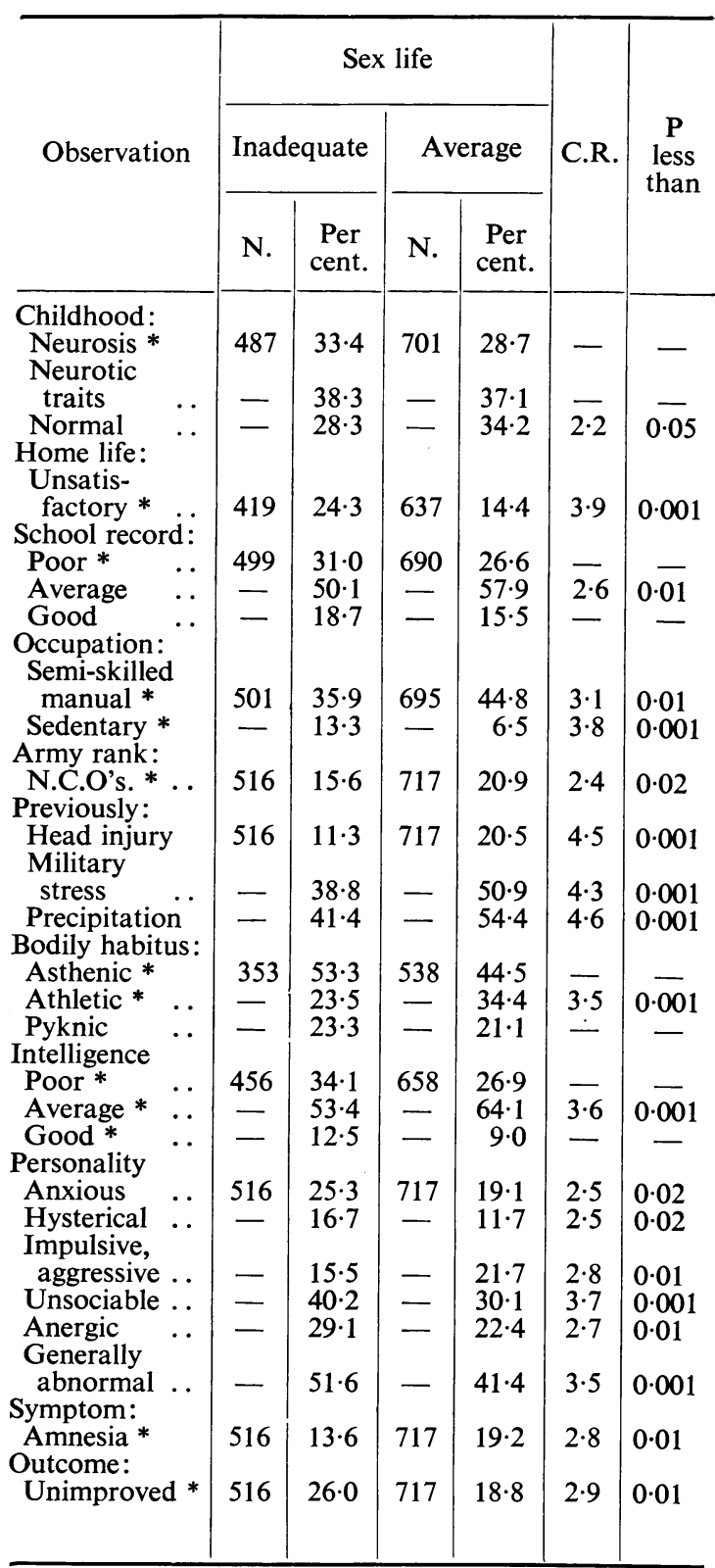

Table showing Frequencies of Various Observations among Neurotic Military Patients, classified by Sex Life. $N$.= number of persons on which relevant observations made.

C.R.=critical ratio, i.e. ration of difference between percentages to its standard error.

$\mathbf{P}$. = probability of such a ratio being obtained by chance. *=comparable finding made when patients classified by degree of military stress undergone (see Table II, Siater, 1943).

The third point is that the table that is published here bears a very remarkable resemblance to Table II in my previous communication (Slater, 1943), where the same clinical material was classified by degree of military stress undergone. All the findings marked by an asterisk in the present table were found to be associated with degree of military stress, and in every case the association is of the 
same kind; i.e. a finding which is more frequent among the sexually inadequate than among the average, is more frequent among those who have not undergone military stress than among those who have. Breakdown in the absence of military stress and inadequate sexuality are themselves positively associated; and it might be supposed that this association might by itself explain their common associations with other psychiatric findings. This suggestion, however, cannot be supported. For, if these associations are expressed in the form of correlation coefficients, the coefficients are all of the order of 0.2 ; that is, they are so low that partial correlation coefficients corrected for their communalities would be very little smaller. The several associations of these two findings, degree of stress undergone and degree of sexual activity, must be regarded as having independent validity.

\section{Discussion}

We must conclude therefore that the factors which predispose to neurotic breakdown are related to those which tend to bring about a reduction of normal sexuality. This is entirely consonant with the hypothesis of Freud. There are, however, other ways of looking at the matter, two of which particularly suggest themselves. We might suppose that the relationship between tendency to neurosis and tendency to sexual inhibition might be in either of two directions. It is possible that the listlessness and inadequacy of personality, which shows itself in so many neurotics, was also expressed to some extent in their sex lives. This would imply that impoverished sexuality was an almost accidental by-product of the general tendency to failure and defeat. On the other hand, it is possible that constitutional factors, such as minor degrees of endocrine insufficiency of various kinds, which result in deficient sexuality, were so impairing the personality as to increase the liability to nervous breakdown. Both hypotheses seem plausible; there is no reason why both should not be to some extent true; and we have no present evidence which would lead us to favour either at the expense of the other. Nevertheless, it may be pointed out that the former hypothesis does not lend itself to translation into any very precise terms, and cannot be subjected to any simple test. The second hypothesis suggests the advisability of investigating the endocrine balance of neurotics, and even provides a hint that the results of such an investigation might eventually prove to be not only of ætiological but also of therapeutic significance.

\section{Summary}

The clinical records of 1,233 neurotic soldiers were classified by the degree of sexual activity they had shown and the frequencies of various psychiatric findings were analysed in the two groups of the sexually inadequate and the sexually average. Sexual inadequacy was found to have significant associations with neurosis in childhood, unsatisfactoriness of home life, school record, occupation, rank in Army, past head injury, degree of military stress undergone, bodily habitus, intelligence, various personality traits, amnesic symptoms, outcome of illness. In nearly every case sexual inadequacy was found to be positively associated with a higher frequency of the abnormal finding. Where a graded characteristic is in question, such as in intelligence and bodily habitus, sexual inadequacy was more frequent at the extremes of the distribution. The findings are closely paralleled by a similar analysis of the same clinical material by degree of military stress undergone. It is concluded that the factors which tend to bring about neurotic breakdown and those which predispose to sexual inadequacy are related. Various hypothetical bases for this observation are discussed. One which appears to be particularly favourable for further investigation suggests that the common factors are to be sought in an imbalance of the endocrine system.

\section{REFERENCES}

Bernard, J. (1935). Human Biol., 7, 430.

Billings, E. G., Ebaugh, F. G., Morgan, D. W., O'Kelly, L. I., Short, G. B., and Golding, F. C. (1943). War Med., 4, 283.

Eysenck, H. J. (1943). Lancet, Sept. 18, 362.

Hill, D. (1944). Proc. roy. Soc. Med., 37, 317.

Rees, W. L., and Eysenck, H. J. (1945). J. ment. Sci., 91, 8 .'

Sargant, W., and Slater, E. (1941). Proc. roy. Soc. Med., 34, 757.

Slater, E. (1943). J. Neurol. Psychiat., 6, 1.

Steinberg, D. L., ar d Wittman, M. P. (1943). War Med., 4, 129. 